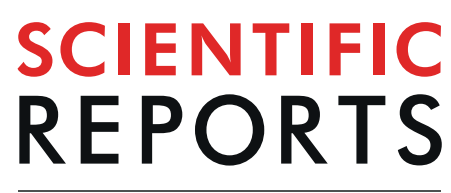

natureresearch

\title{
Normal GCAPs partly compensate for altered cGMP signaling in retinal dystrophies associated with mutations in GUCA1A
}

\begin{abstract}
Daniele Dell'Orco* \& Giuditta Dal Cortivo
Missense mutations in the GUCA1A gene encoding guanylate cyclase-activating protein 1 (GCAP1) are associated with autosomal dominant cone/cone-rod (CORD) dystrophies. The nature of the inheritance pattern implies that a pool of normal GCAP proteins is present in photoreceptors together with the mutated variant. To assess whether human GCAP1 and GCAP2 may similarly regulate the activity of the retinal membrane guanylate cyclase GC-1 (GC-E) in the presence of the recently discovered E111VGCAP1 CORD-variant, we combined biochemical and in silico assays. Surprisingly, human GCAP2 does not activate $\mathrm{GCl}$ over the physiological range of $\mathrm{Ca}^{2+}$ whereas wild-type GCAP1 significantly attenuates the dysregulation of GC1 induced by E111V-GCAP1. Simulation of the phototransduction cascade in a well-characterized murine system, where GCAP2 is able to activate the GC1, suggests that both GCAPs can act in a synergic manner to mitigate the effects of the CORD-mutation. We propose the existence of a species-dependent compensatory mechanism. In murine photoreceptors, slight increases of wild-type GCAPs levels may significantly attenuate the increase in intracellular $\mathrm{Ca}^{2+}$ and cGMP induced by E111VGCAP1 in heterozygous conditions. In humans, however, the excess of wild-type GCAP1 may only partly attenuate the mutant-induced dysregulation of $\mathrm{CGMP}$ signaling due to the lack of GC1-regulation by GCAP2.
\end{abstract}

The absorption of photons by visual pigments in retinal rod and cone cells triggers a complex signaling cascade known as phototransduction, which results in the electrical response of the cell and generates the visual stimulus ${ }^{1}$. A variety of protein-protein, protein-nucleotide and protein-ion interactions finely regulates the cascade ${ }^{2}$, whose dynamics depend on the interplay between $\mathrm{Ca}^{2+}$ and cyclic guanosine monophosphate (cGMP), the second messengers involved in the signaling process ${ }^{3,4}$. The core of the $\mathrm{Ca}^{2+} / \mathrm{cGMP}^{2}$ signaling unit resides in the complex formed by the membrane retinal guanylate cyclase (GC1, also called ROS-GC1 or GC-E being the main cyclase in photoreceptor outer segments ${ }^{5}$ ) and guanylate cyclase-activating proteins (GCAPs). This supramolecular machinery ensures a precise control of the cGMP synthesis by GC1 as a consequence of the level of intracellular free $\mathrm{Ca}^{2+}$, which drops from few hundred nanomolar in the dark to below $100 \mathrm{nM}$ in the light ${ }^{1,6}$. Several GCAP isoforms exist in various species. In mouse $\mathrm{e}^{7}$ and in bovine $\mathrm{e}^{8,9}$ photoreceptors both GCAP1 and GCAP2 have been shown to regulate the target GC1, while in humans so far only GCAP1 has been directly shown to regulate the activity of $\mathrm{GC} 1^{10,11}$.

GCAPs are neuronal calcium sensors that detect subtle changes in $\mathrm{Ca}^{2+}$ concentration and adopt specific conformations required for controlling the activity of the target $\mathrm{GC1}$, per se unable to respond to $\mathrm{Ca}^{2+}$. At high $\left[\mathrm{Ca}^{2+}\right]$, GCAPs adopt a Ca ${ }^{2+}$-loaded state that inhibits GC1 activity, but following the drop in intracellular $\left[\mathrm{Ca}^{2+}\right]$ during the light activation of the cascade, they switch to a $\mathrm{Mg}^{2+}$-bound conformation ${ }^{12-15}$ that stimulates GC1 to rapidly restore dark-adapted cell conditions by enhancing the synthesis of $\mathrm{CGMP}^{3,16}$.

To date, 20 missense mutations have been found in the GUCA1A gene encoding GCAP1, which have been associated with cone (COD) or cone-rod (CORD) dystrophies, severe forms of retinal dystrophy characterized by central vision loss, impaired color vision and photophobia ${ }^{17-19}$. No COD/CORD mutation in the gene encoding GCAP2 is known to date. When studied in reconstituted in vitro systems, most of the point mutations in GUCA1A resulted in GCAP1 variants that constitutively activate the GC1 over the physiological range of $\left[\mathrm{Ca}^{2+}\right]^{10,20-25}$. The 
Y99C-GCAP1 mutation was the first one to have been discovered ${ }^{24}$, moreover it was the first one to be studied in a transgenic mouse line $\mathrm{e}^{26}$. Other transgenic mouse lines were then generated to study similar COD/CORD-related phenotypes associated with mutations in GCAP1, namely the E155G-GCAP $1^{27}$ and L151F-GCAP $1^{28}$ models. The in vivo studies showed that the COD-related mutant caused photoreceptor degeneration due to an elevated $\left[\mathrm{Ca}^{2+}\right]$ in the rod outer segment. However, rod photoresponses from the Y99C-GCAP1 mice showed relatively little changes especially at bright flashes, indicating a partly preserved $\mathrm{Ca}^{2+}$-regulated cGMP synthesis. Interestingly, the rate of photoreceptor cell loss increased with the level of Y99C-GCAP1 expression ${ }^{26}$.

For bovine and murine photoreceptors, it is well established that GCAP1 and GCAP2 are both capable of activating and inhibiting the target GC1 at similar levels in the physiological range of $\left[\mathrm{Ca}^{2+}\right]$, however the two GCAPs differ from one another in some features: (i) GCAP1 has a slightly lower affinity for $\mathrm{Ca}^{2+}$ compared to GCAP2 ${ }^{12}$; (ii) GCAP1 triggers the activation of GC1 earlier than GCAP2, that is at dimmer light conditions and/ or when intracellular $\mathrm{Ca}^{2+}$ is still relatively high ${ }^{3}$; (iii) both GCAPs are myristoylated, but the myristoylation

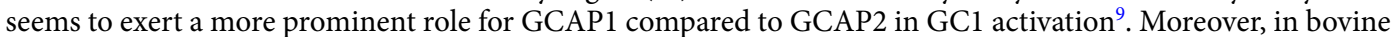
cells the cellular concentration of GCAP1 and GCAP2 sums to the cellular concentration of GC1 ${ }^{9}$. While it is clear that both GCAPs can regulate GC1 over the narrow physiological window of intracellular $\mathrm{Ca}^{2+}$, quite different mechanisms have been proposed for the regulation. Some studies support distinct binding interfaces between GC1 and GCAP1/GCAP2, the first GCAP being located at the juxtamembrane region and the second prevalently bound to the catalytic- $C$ terminal domain ${ }^{29-31}$, thus allowing the binding of GCAP1 and GCAP2 to either the same or distinct GC1 molecules, but always in different interfaces On the other hand, other studies found a partly overlapped GC interface for GCAP1 and GCAP2 ${ }^{32,33}$, suggesting a competition between GCAP1 and GCAP2 for $\mathrm{GC1}$ activation in mouse cones ${ }^{34}$ as well as in rods. Overall, GCAPs seem to operate in a "calcium-relay" mode, thus acting simultaneously on either the same or distinct GC1 molecules to induce gradual responses in terms of cGMP synthesis triggered by small changes in intracellular $\left[\mathrm{Ca}^{2+}\right]^{3,35}$. Their slightly different GC1-regulatory properties and $\mathrm{Ca}^{2+}$-sensitivity would therefore be needed to achieve the complex temporal dynamics necessary for finely tuning the enzymatic activity of GC1 over the broad range of light stimuli typical of photoreceptors.

COD and CORD linked to mutations in GUCA1A are associated with autosomal dominant (ad) inheritance pattern, thus implying that one half of the GCAP1 molecules in the overall GCAP pool would carry the mutation in affected photoreceptors. Under the assumption that no other alteration beside the missense mutation occurs, a normal amount of GCAP2 molecules would be therefore still present, together with one half of normal GCAP1. In this work we present the results of biochemical assays of human GC1 catalytic activity performed with different amounts of human wild-type (WT)-GCAP1, GCAP2 and E111V-GCAP1, a recently discovered variant associated with a severe form of $\mathrm{CORD}^{10}$. Interestingly, we found that human GCAP2 is uncapable of activating GC1 and therefore does neither co-act nor compete with GCAP1. On the other hand, increasing the amount of WT-GCAP1 with respect to E111V-GCAP1 led to a partial restoration of the $\mathrm{Ca}^{2+}$-regulation of GC1 enzymatic activity, although a 3-fold excess of WT was not enough to restore a fully normal behavior. In order to predict the dysregulation of the $\mathrm{Ca}^{2+}$ and cGMP homeostasis in a rod cell under conditions mimicking different expression levels of mutated GCAP1, we transferred the quantitative results from the enzymatic assays to a comprehensive model of mouse phototransduction that showed capable of reproducing photoresponses under both dim and bright stimuli ${ }^{36,37}$. We observed a crucial role for GCAP2 in mouse in compensating the dysregulation induced by the disease-associated E111V-GCAP1 which cannot possibly occur in human cones due to the lack of activity on GC1.

\section{Results and Discussion}

Regulation of the GC1 activity by $\mathrm{Ca}^{2+}$ via GCAPs represents an exemplary case, in which an enzyme (GC1) that per se is not sensitive to $\mathrm{Ca}^{2+}$ can be either inhibited or activated by the same molecule (GCAP) depending on the subtle changes in intracellular $\left[\mathrm{Ca}^{2+}\right]$. The discovery of the highly cooperative negative feedback mechanism mediated by $\mathrm{Ca}^{2+}$ on the GC1 dates back to the late $80 \mathrm{~s}^{38}$, and yet the fine mechanisms of its regulation are not completely understood. It has been established that the GCAP1/2-mediated $\mathrm{Ca}^{2+}$ feedback on GC1 activity is the only one that occurs at very dim light intensities, corresponding to the single photon response ${ }^{39}$. By integrating quantitative information arising from assays performed with recombinant systems and numerical simulations, we sought to clarify some mechanisms that are apparently perturbed in cases such as the severe CORD form recently associated with the E $111 \mathrm{~V}$ mutation in GCAP $1^{10}$. To investigate the role of each GCAP variant, namely WT-GCAP1, E111V-GCAP1 and WT-GCAP2 in GC1 (dys)regulation, we performed biochemical assays both in the presence of individual GCAPs and in the co-presence of the variants, and measured quantitative parameters to describe the $\mathrm{Ca}^{2+}$-dependence of the cGMP synthesis by GC1 and the apparent cooperativity of the process.

Human GCAP2 does not activate human GC1. Human GCAP1 (both WT and carrying the E1111V mutation) and GCAP2 were heterologously expressed and purified at high purity levels. All GCAP variants responded to $\mathrm{Ca}^{2+}$ by switching to a different conformation that showed the typical increase in electrophoretic mobility (Fig. 1a), as observed in other calcium sensors ${ }^{40}$. The presence of smeared or multiple bands in SDS-PAGE gels is usual for calcium sensor proteins, and is related to the residual capability of the protein to bind $\mathrm{Ca}^{2+}$ in spite of the detergent-induced denaturation and the chelator present in the buffer ${ }^{12}$. Human GC1 was stably expressed in HEK cells and it concentrated in the membrane milieu (Fig. S1b), thus allowing the correct in vitro reconstitution of the GCAP-GC1 complex for biochemical assays.

While WT-GCAP1 was able to activate GC1 at low $\mathrm{Ca}^{2+}$, as observed in previous studies ${ }^{10,11}$, GCAP2 was unable to exert any regulation of GC1 as in both low and high $\mathrm{Ca}^{2+}$ conditions the levels of cGMP synthesis were unaltered with respect to those of the control (Fig. 1b). When the enzymatic assays were performed with equal amounts of GCAP1 and GCAP2, at low $\mathrm{Ca}^{2+}$ a slight decrease in the GC1 activation was observed $(n=3$, $\mathrm{p}=0.045$ ) with respect to the sole presence of GCAP1, thus indicating that the presence of GCAP2 only slightly 

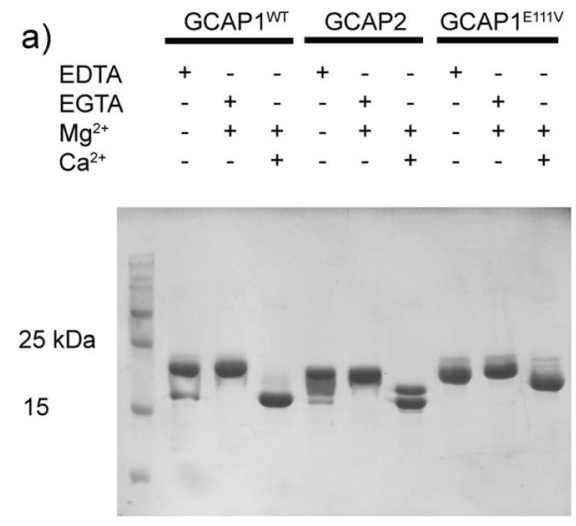

b)

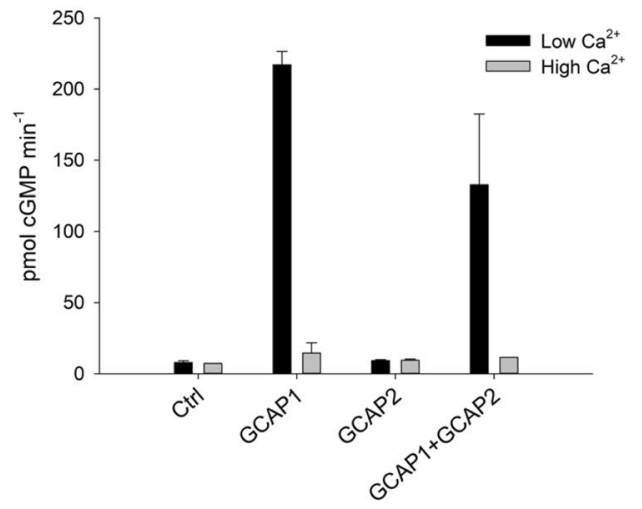

Figure 1. Purity of recombinant proteins, $\mathrm{Ca}^{2+}$-induced gel shifts and $\mathrm{GC} 1$ enzymatic assays. (a) SDS-PAGE of WT/E111V GCAP1 and GCAP2 in the presence of $5 \mathrm{mM}$ EDTA, $4 \mathrm{mM}$ EGTA $+1.4 \mathrm{mM} \mathrm{Mg} \mathrm{Mg}^{2+}$ and $1 \mathrm{mM}$ $\mathrm{Mg}^{2+}+4 \mathrm{mM} \mathrm{Ca}^{2+}$. Twenty micromolar WT-GCAP1, E111V-GCAP1 and WT-GCAP2 were incubated for $10 \mathrm{~min}$ at $30^{\circ} \mathrm{C}$ in the presence or absence of ions and loaded in a 15\% SDS-PAGE. Gel was Coomassie bluestained. (b) GC1 enzymatic assays in the presence of $10 \mu \mathrm{M}$ GCAP1, $10 \mu \mathrm{M}$ GCAP2 and equal amounts of both $(5 \mu \mathrm{M}$ GCAP $1+5 \mu \mathrm{M}$ GCAP2 $)$ in the presence of less than $19 \mathrm{nM} \mathrm{Ca}^{2+}\left(\right.$ low Ca $\left.{ }^{2+}\right)$ or $\sim 30 \mu \mathrm{M} \mathrm{Ca}^{2+}\left(\right.$ high Ca$\left.^{2+}\right)$. Each bar represents the average of three replicas \pm standard deviations $(n=3)$. Full-length gels are reported in Supplementary Fig. S1.

interferes with the activity of GCAP1, which was still capable of regulating GC1. No GCAP2-induced activation of GC1 was observed even when the experiments were performed at higher (12 mM) $\mathrm{Mg}^{2+}$ (Fig. S2), thus indicating that the inability is intrinsic and not due to a low amount of $\mathrm{Mg}^{2+}$-bound GCAP2 in the assay.

The finding that human GCAP2 does not activate human GC1 might seem surprising, but in fact it is in line with previous work indicating that in human retinas, and similarly in monkey, GCAP2 localizes in cone inner segments, soma and synaptic terminal and only at very low levels in inner segments ${ }^{41}$. GCAP2 is crucial for maintaining the integrity of the photoreceptor synaptic terminal, likely mediating the effect of light on the morphological remodeling changes of synaptic ribbons ${ }^{42}$ and even a single phosphorylation of GCAP2 has been shown to cause the complete retention of the protein in the inner segment ${ }^{43}$. Thus, while experiments with GCAPs ${ }^{-1-}$ transgenic mice clearly established a role for GCAP2 in regulating the GCAP1-dominated GC1 activity ${ }^{39,44}$, and thorough in vitro investigations confirmed such capability ${ }^{7}$, in humans GCAP2 might have not-yet clear functions non-related with phototransduction.

CORD-associated E111V-GCAP1 is dominant and leads to GC1-dysregulation. Since GCAP2 did not contribute to the activation of human GC1, we monitored the $\mathrm{Ca}^{2+}$-dependent regulation of the cyclase in the co-presence of different amounts of WT-GCAP1 and E111V-GCAP1, with the goal to quantitatively assess the effects of the two variants on the same enzymatic target. Over the relatively narrow window of physiological variations of intracellular $\left[\mathrm{Ca}^{2+}\right](\sim 10 \mathrm{nM}-600 \mathrm{nM})$, WT-GCAP1 correctly switched from GC1-inhibitor to GC1-activator (Fig. 2, black curve). The $\mathrm{IC}_{50}$ value, i.e. the $\left[\mathrm{Ca}^{2+}\right]$ at which the synthesis of cGMP and therefore the activity of GC was half-maximal, was $251 \pm 19 \mathrm{nM}(\mathrm{n}=6)$ and the cooperativity of the process was high $\left(\mathrm{h}_{\mathrm{c}}=2.60 \pm 0.42 ; \mathrm{n}=6\right.$; Table 1$)$.

Like many other COD/CORD-associated GCAP1 mutants, E111V-GCAP1 showed a dramatic shift of GC1 regulation to very high $\mathrm{Ca}^{2+}$ levels, with $\mathrm{IC}_{50}{ }^{\mathrm{E} 111 \mathrm{~V}}=10 \pm 5 \mu \mathrm{M}(\mathrm{n}=4)$ and a significantly lower cooperativity $\left(\mathrm{h}_{\mathrm{c}}=0.83 \pm 0.27, \mathrm{n}=4 \text {; see red curve in Fig. } 2\right)^{10}$ compared to the WT, thus constitutively activating the GC1 target over a physiological range of $\left[\mathrm{Ca}^{2+}\right]$. Those biochemical assays, however, were performed in the sole presence of E111V-GCAP1. When the assay was performed by mixing equal amounts of WT and E111V-GCAP1, thus resembling the heterozygous $\mathrm{WT} / \mathrm{E} 111 \mathrm{~V}$ case found in CORD patients, the situation led to a different scenario (Fig. 2, green curve). While no significant change in the cooperativity could be observed (Table 1), the $\mathrm{IC}_{50}$ value shifted down to $694 \pm 75 \mathrm{nM}(\mathrm{n}=6)$ (Fig. 2, Table 1). Although this value is still insufficient to inhibit the GC1 at physiologically high $\left[\mathrm{Ca}^{2+}\right]$, leading to $\sim 55 \%$ of the maximal activity, it is definitely lower compared to the $\sim 90 \%$ activity of E111V-GCAP1 alone.

WT-GCAP1 is thus capable of partly restoring the functional switch of GC1 in the presence of a mutant that would otherwise block the enzyme in a constitutively active state. A similar result was previously obtained by Dizhoor and co-workers ${ }^{21}$, who investigated in a bovine system the Y99C-GCAP1 variant associated with COD, and noticed that enzymatic assays in the co-presence of WT and mutated GCAP1 still led to constitutive GC1 activity. The authors also investigated the co-presence of Y99C-GCAP1 and WT-GCAP2, obtaining very similar results, and thus concluded that the mutant stimulates the cyclase in the presence of equimolar concentrations of 


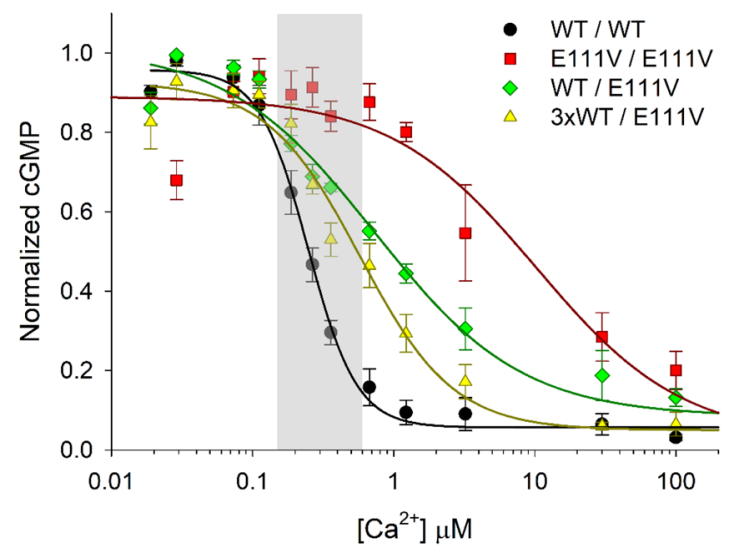

Figure 2. $\mathrm{Ca}^{2+}$-dependence of GC1 activity in the presence of various GCAP1 variants. The GC1 activity as function of $\left[\mathrm{Ca}^{2+}\right]$ was measured in the presence of $10 \mu \mathrm{M}$ WT-GCAP1 (black circles), $5 \mu \mathrm{M}$ WTGCAP1 + 5 $\mu \mathrm{M}$ E111V-GCAP1 (green diamonds) and 15 $\mu \mathrm{M}$ WT-GCAP1 $+5 \mu \mathrm{M}$ E111V-GCAP1 (yellow triangles). E111V-GCAP1's activity profile is referred to the published data ${ }^{10}$. Each data set is relative to $4-6$ replicas and data are normalized according to both total protein content in membranes and maximum and minimum GC1 activity recorded in each replica; error bars represent s.e.m. Solid lines represent the results of data fitting while the grey box represents the physiological $\mathrm{Ca}^{2+}$ fluctuations in rod photoreceptors.

\begin{tabular}{|l|l|l|l|l|}
\hline & GCAP1 $^{\text {WT }}(\mathbf{n}=\mathbf{6})$ & GCAP1 $^{\text {EIIIV }}(\mathbf{n}=\mathbf{4})$ & GCAP1 $^{\text {WT/E111V }}(\mathbf{n}=\mathbf{6})$ & 3XGCAP1 $^{\text {WT }} /$ GCAP1 $^{\text {EIIIV }}(\mathbf{n}=\mathbf{6})$ \\
\hline $\mathrm{IC}_{50}(\mathrm{nM})$ & $251 \pm 19^{\mathrm{a}}$ & $(10 \pm 5) \times 10^{3}$ & $694 \pm 75$ & $497 \pm 49$ \\
\hline $\mathrm{h}_{\mathrm{c}}$ & $2.60 \pm 0.42$ & $0.83 \pm 0.27$ & $0.87 \pm 0.18$ & $1.49 \pm 0.35$ \\
\hline
\end{tabular}

Table 1. Results from enzymatic assays. Human GC1 activity as a function of free $\left[\mathrm{Ca}^{2+}\right]{ }^{\text {(a) }}$ reported data are mean \pm s.e.m. ${ }^{\text {(b) }}$ data from. ref. ${ }^{10}$.

either WT-GCAP1 or GCAP2 ${ }^{21}$. However, those assays were performed in a bovine system, where GCAP2 plays a role in $\mathrm{GC1}$ activation, at odds with our current findings referring to a human system.

Since human GCAP2 does not activate GC1, we sought to investigate whether the observed partial restoring capability of WT-GCAP1 to restore GC1 activity in the presence of E111V-GCAP1 could even increase in the presence of extra WT-GCAP1. The assays were performed with 3-fold excess of WT-GCAP1, while keeping the concentration of E111V unaltered with respect to the previous conditions. Interestingly, a statistically significant decrease of $\mathrm{IC}_{50}$ was observed ( $497 \pm 49$ compared to $694 \pm 75 ; \mathrm{n}=6$; one-tailed $\mathrm{p}=0.029$; Table 1$)$. Although the residual activity of $\mathrm{GCl}$ at physiologically high $\left[\mathrm{Ca}^{2+}\right]$ was still quite high ( $\sim 45 \%$; Fig. 1, yellow curve) the regulation profile became more cooperative, as shown by the increase in $h_{c}(1.49 \pm 0.35$ vs. $0.87 \pm 0.18, n=6)$. Therefore, although the switch was still non-optimal, the presence of 3-fold excess WT-GCAPs seems to slightly attenuate the dominant and detrimental effect caused by the E111V-GCAP1 mutation.

The observed results of GC1 activity in the presence of different amounts of GCAP1 and its E111V variant are not straightforward to interpret. Human WT-GCAP1 and E111V-GCAP1 have very similar apparent affinity $\left(\mathrm{EC}_{50}\right)$ for $\mathrm{GC1}^{10}$, therefore it seems unlikely that the cause of the still dominant effect of the mutant in a situation of 3-fold excess of WT be attributed to the preferential formation of a complex with the GC1. A more realistic explanation could be the existence of different equilibria between the oligomeric states of WT-GCAP1 and E111V-GCAP1, which could result in different amounts of monomeric GCAP available for GC regulation. GCAP1 is known to form dimers ${ }^{10,45}$ and under conditions mimicking the physiological ones E111V-GCAP1 was also found to be dimeric ${ }^{10}$. We cannot exclude that in the conditions of our in vitro assays the tendency of WT-GCAP1 to form dimers enhanced at the higher concentrations corresponding to the 3 -fold excess experiments, de facto limiting the availability of monomers that could further improve the restoration of the GC1 regulation towards a physiological behavior.

Expression levels of normal GCAPs set the homeostasis of $\mathrm{Ca}^{2+}$ and $\mathrm{cGMP}$ in a computational model of E111V-GCAP1 mouse rod. In order to evaluate the putative cellular consequences of the $\mathrm{Ca}^{2+} /$ cGMP dysregulation brought about by the E111V-GCAP1 mutation associated with CORD and the extent of a potential compensation by normal GCAPs, we used the parameters experimentally measured in the human system to predict the rate of cGMP synthesis in a mouse rod illuminated by flashes of increasing intensity. We used a comprehensive kinetic model of phototransduction that demonstrated very effective in reproducing experimental results from dim to bright light conditions ${ }^{37}$. In particular, we could directly probe the role of each GCAP, namely WT/E111V GCAP1 and GCAP2, in regulating the levels of second messengers by virtually tuning the contribution of each protein to GC1 regulation and simulating the dark-adapted state of the cell as well as the response to specific light stimuli, thus investigating the dynamic shaping of cGMP synthesis. 


\begin{tabular}{|l|l|l|l|l|}
\hline & GCAP1 $^{\text {WT/E111Va }}$ & GCAP1 $^{\text {E111V/E111V }}$ & 3XGCAP1 $^{\text {WT }} /$ GCAP1 $^{\text {E111V }}$ +GCAP2 & 3XGCAP1 $^{\text {WT }}$ /GCAP1 $^{\text {E111V-GCAP2 }}$ \\
\hline X-fold $\left[\mathrm{Ca}^{2+}{ }_{\text {free }}\right]$ & 3.1 & 12.5 & 1.4 & 3.0 \\
\hline X-fold [cGMP] & 1.4 & 2.0 & 1.1 & 1.4 \\
\hline
\end{tabular}

Table 2. Increase in dark levels of $\mathrm{Ca}^{2+}$ and cGMP according to numerical simulations of a mouse rod outer segment compared to a wild-type case. ${ }^{a}$ combination of individual weight factor $\mathrm{f}_{\mathrm{i}}$ to simulate each condition are illustrated in the Methods.

The significant perturbation of the GC1 regulation induced by the E111V mutation in GCAP1 assessed in vitro was reflected by a substantial increase of the dark levels of $\mathrm{Ca}^{2+}$ and cGMP (Table 2).

The simulation of the rod in the dark for a heterozygous WT/E111V mouse resembles the case of adCORD pattern in humans, in which half of the GCAP1 pool is made of WT molecules and the other half carries the E111V mutation. Under these conditions, a 3.1-fold increase in the level of intracellular $\mathrm{Ca}^{2+}$ and 1.4 -fold increase in that of cGMP was predicted by numerical simulations (Table 2). These results are substantially in line with the experimental measurements of intracellular $\left[\mathrm{Ca}^{2+}\right]$ in Y99C-GCAP1 mice, where an increase of 1 to 2 -fold depending on the expression levels of the mutant over a background of WT-GCAP1 was observed ${ }^{26}$. To probe the effects of different expression levels of mutant vs. WT-GCAP1, we performed numerical simulations of other putative cases, first one in which the whole pool of GCAP1 carried the E111V mutation in the co-presence of endogenous WT-GCAP2. The simulated increase of dark levels of $\mathrm{Ca}^{2+}$ and cGMP was dramatic in this hypothetical E111V/E111V homozygosis case (12.5-fold and 2.0-fold, respectively; Table 2). In line with our in vitro experiments with human proteins, we also simulated the effects of a 3-fold excess of WT-GCAP1 over a background of constant E111V-GCAP1, both in the presence and in the absence of endogenous GCAP2. Interestingly, extra-delivery of WT-GCAP1 in the presence of GCAP2 restored an almost WT-like homeostasis of dark Ca ${ }^{2+}$ and cGMP (1.4-fold and 1.1. fold, respectively; Table 2). However, the same delivery of WT-GCAP1 performed in the absence of endogenous GCAP2 did not restore a WT-like homeostasis for second messengers, and led to a dramatic increase of 3.0-fold dark $\mathrm{Ca}^{2+}$ and 1.4-fold cGMP, similar to what was observed in the simulated heterozygous WT/E111V case (Table 2). Hence, simulations suggest that GC1 activation by GCAP2 is fundamental in mouse photoreceptor to set the normal levels of second messengers. In its absence, the normally dominant WT-GCAP1 although delivered in excess cannot compensate for the detrimental dysregulation of GC1 by the pathogenic E111V mutation.

GCAP2 shapes CGMP synthesis in E111V-GCAP1 mouse photoresponses. Perturbation of the second messenger homeostasis in the dark might lead to alterations in the photoresponse of the affected cells. To probe such potential variations, we simulated photoresponses from mouse rods expressing the same amounts of GCAPs investigated in the dark following the excitation of the phototransduction cascade by flashes of increasing intensity, from dim to saturating. The resulting dynamics of suppression of the dark current in a rod of a WT mouse and the respective cases for a heterozygous WT/E111V and a homozygous E111V/E111V-GCAP1 case are reported in Fig. 3a,b, respectively.

For all the tested flash intensities the photoresponses of the mutant cases were prolonged compared to the WT, with increased time to peak. The prolongation of the photoresponse apparently depends on the relative abundance of E111V-GCAP1 with respect to the WT and was in fact significantly more pronounced in the homozygous (Fig. 3b) compared to the heterozygous case (Fig. 3a). Similar results were experimentally measured in Y99C-GCAP1 mice ${ }^{26}$, although the effect was less prominent, probably due to the milder phenotype that developed COD and not CORD as in the case of E111V-GCAP1.

Our computational implementation of the GC1 regulation by different GCAP variants allowed us to ideally dissect their individual contributions in shaping the rate of cGMP synthesis at different light stimuli. Figure 3c,d reports on the overall GCAPs contribution (black line) to the time course of cGMP synthesis as a sum of the contribution by GCAP1 variants (blue line) and WT-GCAP2 (red line). Clear differences could be observed between $\mathrm{WT}$ and WT/E111V heterozygous case. For WT rods, increasing the flash intensity led to an increasing contribution of GCAP2 in shaping the cGMP synthesis rate, which was less important for very dim flashes, but became predominant at bright intensities (Fig. 3c), in line with the $\mathrm{Ca}^{2+}$-relay mechanism ${ }^{3,35}$. In the case of WT/E111V heterozygous mouse, however, the unbalance in the contributions became apparent under dim light conditions, where GCAP1 variants dominated, while GCAP2 was still capable of shaping the rate at brighter flashes (Fig. 3d). It should be noticed that, in spite of the dramatic perturbation in the dark levels of $\mathrm{Ca}^{2+}$ ad cGMP (Table 2), our model predicts that the photoreceptor would still respond to light stimuli, with all in all minor perturbation of the photoresponse shape (Fig. 3a), thus fully in line with experimental observations ${ }^{26}$.

The major dysregulation of second messenger homeostasis observed in the hypothetical E111V/E111V homozygous case, where the whole GCAP1 pool carried the E111V mutation reflected in a dramatic perturbation of the photocurrent shapes, which became significantly prolonged and did not completely shut-off even after $5 \mathrm{~s}$ for brighter stimuli (Fig. 3b). Under dim flashes, GCAP2 did not contribute at all to the shaping of the cGMP synthesis rate, but at middle to bright light conditions, where GCAP1 was almost completely blocked in the GC1-constitutively activating state, GCAP2 provided most of the dynamic contribution (Fig. 3e).

Simulation of 3-fold WT-GCAP1 delivery over a background of E111V-GCAP1 variant suggested a scenario that strictly depends on the presence of the other endogenous GCAP2. Indeed, when endogenous GCAP2 was considered in the simulations, the mutant-induced prolongation of the photocurrent was reduced at all light intensities (Fig. 4a) and the dynamics of cGMP synthesis as well as the specific contributions of GCAPs (Fig. 4c) 
WT/E111V

a)

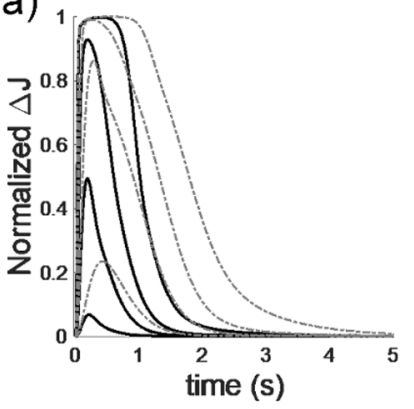

E111V/E111V

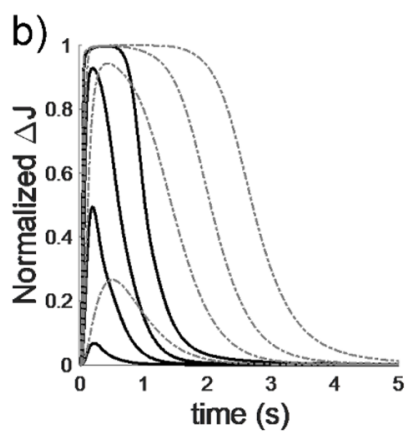

c) $1.54 R^{*}$

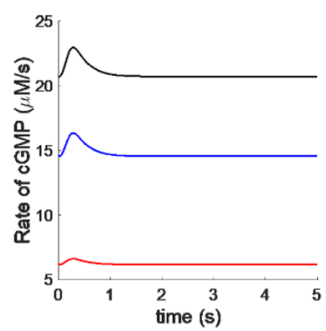

d)

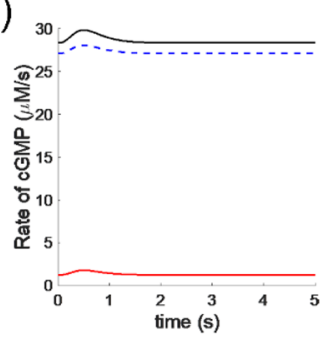

e)

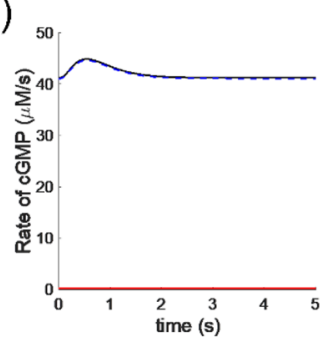

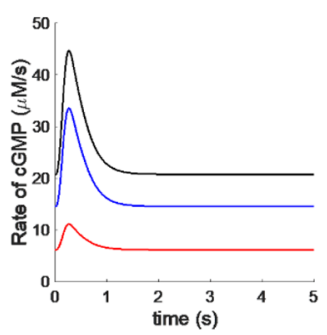
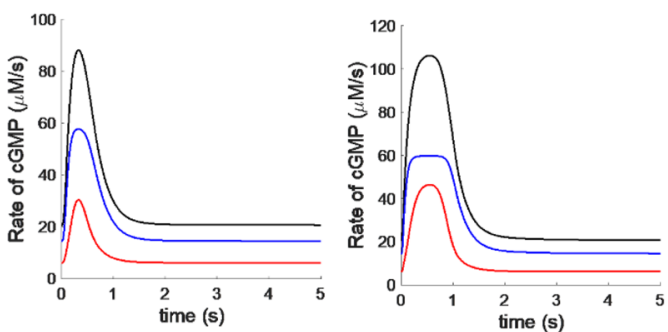

WT/E111V
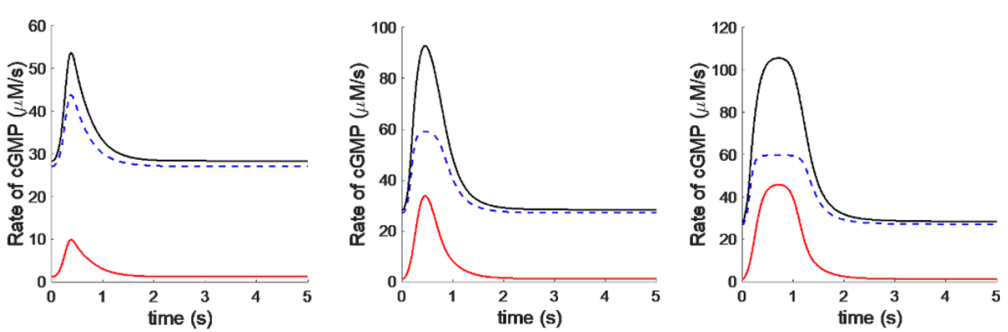

\section{E111V/E111V}
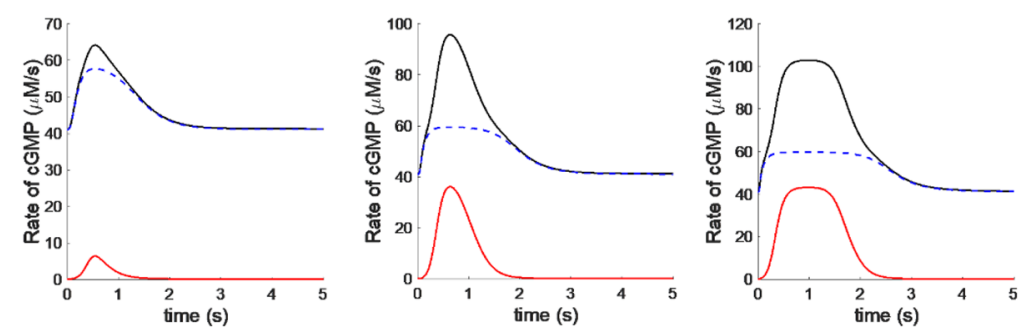

Figure 3. Simulation of flash responses and dynamics of cGMP synthesis in a mouse rod, from dim to bright light conditions. After equilibration of the rod outer segment in the dark, $24 \mathrm{~ms}$ flashes were delivered, leading to $1.54,18,87$ and 500 photoisomerizations of rhodopsin; (a,b) Black traces: WT GCAPs-containing rods.

Dash-dotted gray lines: photoresponses in a WT/E111V heterozygous case (a) and E111V/E111V homozygous case (b). Photocurrents represent the suppression of the dark current at each light intensity and have been normalized. (c-e) Simulated time course of the rate of cGMP synthesis by GC1 in the same conditions as in $(\mathbf{a}, \mathbf{b})$. Blue lines: contribution by WT-GCAP1 + E111V-GCAP1 (if present). Red lines: contribution by GCAP2. Black lines: overall GCAPs contribution obtained by adding up the former terms.

were overall fairly similar to the WT case (Fig. 3c). This suggests that under these conditions it would be in principle possible to partly restore a normal shaping of both the photocurrent and cGMP synthesis profile, thus minimizing the perturbation of the second messengers (Table 2). However, when the same delivery of 3-fold WT-GCAP1 was simulated in the absence of endogenous GCAP2, the prolongation of the photocurrents became significant under all light regimes (Fig. 4b), resembling that of heterozygous WT/E111V (Fig. 3a). Nevertheless, in this case the absence of GCAP2 led to a completely GCAP1 (WT and E111V)-driven cGMP synthesis, with 
a) 3xWT/E111V

(+GCAP2)

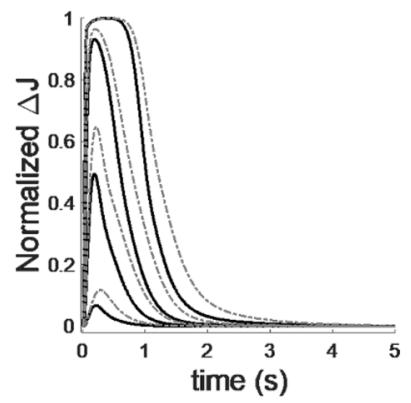

b) 3xWT/E111V

(-GCAP2)

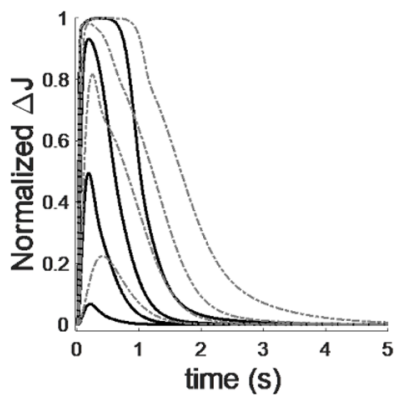

3xWT/E111V (+GCAP2)

c)

\section{$1.54 \mathrm{R}^{*}$}

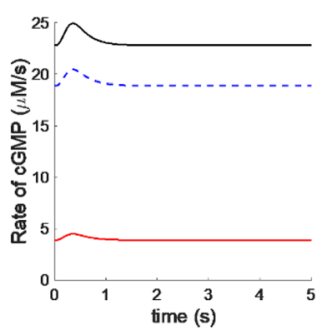

d)

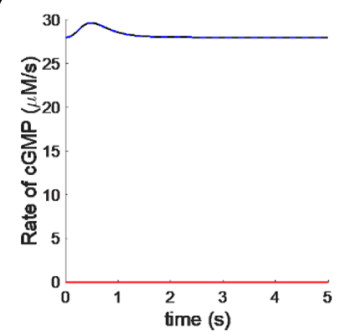

$18 \mathrm{R}^{*}$

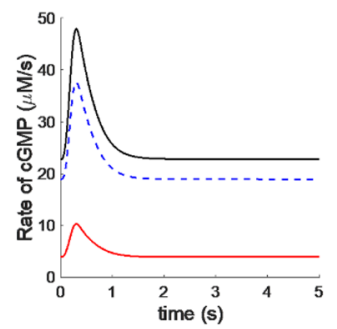

$87 \mathrm{R}^{*}$
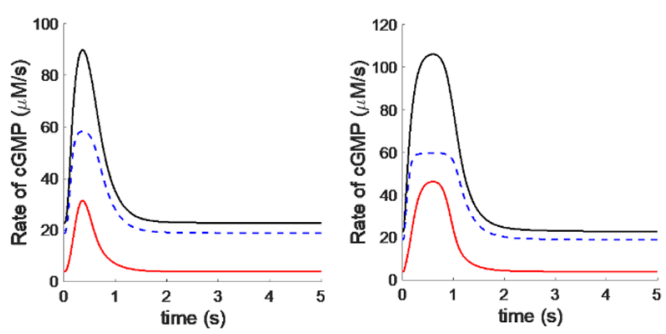

3xWT/E111V (-GCAP2)
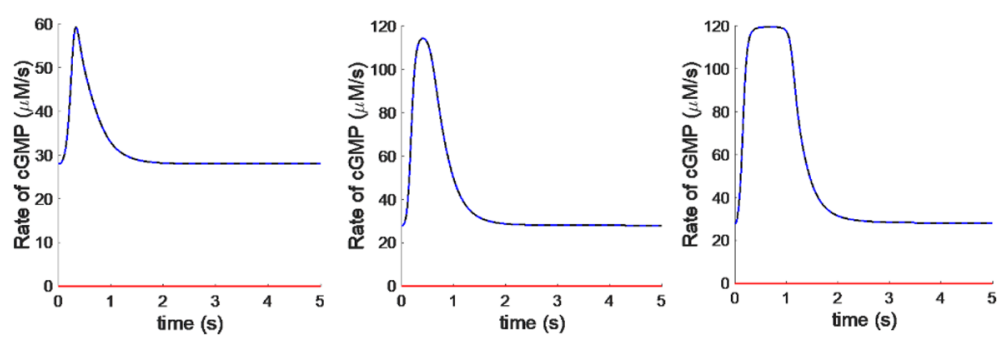

Figure 4. Simulation of flash responses and dynamics of cGMP synthesis in a mouse rod, from dim to bright light conditions, in the presence of 3-fold WT-GCAP1 with respect to E111V-GCAP1. Panels $(\mathbf{a}, \mathbf{c})$ refer to simulations in which endogenous GCAP2 was present. Panels $(\mathbf{b}, \mathbf{d})$ refer to simulations in the absence of endogenous GCAP2. All simulated conditions and symbols are the same of Fig. 3.

higher maximal levels of synthesis at each light intensity (Fig. 4d) compared to the heterozygous case (Fig. 3d). Hence, in spite of the dominant role of this GCAP1 isoform in the WT photoreceptor, the excess of GCAP1 alone is not sufficient to compensate for the absence of GCAP2 in mouse rods.

The crucial role of GCAP2 in compensating for the extreme perturbations of cGMP signaling induced by blocking GCAP1 into a GC1-constitutively active state was proposed by us for a bovine system based on comprehensive model of the phototransduction kinetics ${ }^{46}$ and was then experimentally confirmed in a mouse cone system $^{34}$.

\section{Conclusions}

In conclusion, our findings support the view that the severity of COD/CORD phenotypes associated with point mutations in GUCA1A correlates both with the dysregulation of the CGMP signaling induced by the point mutation and with the levels of mutated GCAP1 over WT-GCAPs. Increasing the levels of WT-GCAP1 on a background of disease-associated mutants may partly attenuate the increased dark levels of $\mathrm{Ca}^{2+}$ and cGMP, which are ultimately associated to cell death ${ }^{47,48}$ and reduce the perturbation of the photocurrent dynamics. Since promising strategies for the delivery of WT recombinant proteins to photoreceptors are under development ${ }^{49,50}$, our findings might be relevant for future protein therapies aimed at slowing retinal degenerations associated with GUCA1A mutants. However, our data also show that, while useful for the purpose, extra-WT-GCAP1 alone would probably not be sufficient to restore a normal regulation of GC1 in human retinas. The intricate $\mathrm{Ca}^{2+}$-relay mechanism involving GC1 and the GCAPs might be substantially species-dependent, and whether other regulators in human photoreceptors may mimic the synergic activation of GCAP1 and GCAP2 on GC1 observed in mouse and bovine retinas remains to be clarified. 


\section{Materials and Methods}

GCAPs expression and purification. Synthetic genes (Genscript) corresponding to the cDNA of human GCAP1 (Uniprot: P43080) and GCAP2 (Uniprot: Q9UMX6) were cloned into pET-11a(+) vectors using NdeI and NheI as restriction site. Mutagenesis, protein expression and purification of myristoylated proteins were performed as described previously ${ }^{10,12}$. Briefly, cells were let grown at $37^{\circ} \mathrm{C}$ until an $\mathrm{OD}_{600}$ value of 0.4 was reached, then myristic acid $(100 \mu \mathrm{g} / \mathrm{mL}$, in $50 \% \mathrm{EtOH}, \mathrm{pH} 7.5)$ was added. After the induction with $1 \mathrm{mM}$ IPTG, cells were let grown for 4 hours at $37^{\circ} \mathrm{C}$. Both WT and E111V-GCAP1 as well as GCAP2 were purified from inclusion bodies. After protein denaturation by $6 \mathrm{M}$ guanidinium hydrochloride and refolding steps by dialysis, two subsequent chromatographic steps (size exclusion and anionic exchange chromatography) were performed. Proteins purity was tested by Polyacrylamide Gel Electrophoresis in the presence of Sodium-Dodecyl-Sulfate (SDS-PAGE) and was found to be at least 95\% (see Fig. 1a). The efficiency of protein myristoylation was proven by HPLC and mass spectrometry. After buffer exchange ( $20 \mathrm{mM}$ TRIS, $150 \mathrm{mM} \mathrm{NaCl}, 1 \mathrm{mM}$ DTT), proteins were shock frozen and stored at $-80^{\circ} \mathrm{C}$ until use.

GC expression and enzymatic assays. A stable cell line expressing human guanylate cyclase isoform 1 (GC1) was obtained as explained previously ${ }^{10,51}$ by transfecting HEK293 flp cells with pIRES-eGFP plasmid using Turbofect reagent. Transfected clones were selected based on the resistance to geneticin (G418). Cells were cultured in DMEM media containing fetal bovine serum $(10 \% \mathrm{v} / \mathrm{v})$, streptomycin $(100 \mu \mathrm{g} / \mathrm{mL})$, penicillin $(100$ units $/ \mathrm{mL}$ ) and geneticin $(500 \mu \mathrm{g} / \mathrm{mL})$. At $90 \%$ confluence the cells were harvested, washed with sterile PBS and stored at $-80^{\circ} \mathrm{C}$. To perform enzymatic assays on isolated membranes cells pellets were suspended in lysis buffer (10 mM HEPES pH 7.4, $1 \mathrm{mM}$ DTT, protease inhibitor cocktail 1X) and incubated for $20 \mathrm{~min}$ on ice. After 15 up-and-down cycles with a $1 \mathrm{~mL}$ syringe on ice, cells were centrifuged for $20 \mathrm{~min}$ at $10000 \mathrm{rpm}$. The obtained pellets were suspended in $50 \mathrm{mM}$ HEPES pH 7.4, $50 \mathrm{mM} \mathrm{KCl}, 20 \mathrm{mM} \mathrm{NaCl}, 1 \mathrm{mM}$ DTT.

First, GC1 activity was assessed incubating $10 \mu \mathrm{M}$ GCAP1 or GCAP2 or a combination of both $(5 \mu \mathrm{M}$ GCAP1 $+5 \mu \mathrm{M}$ GCAP2 $)$ in the absence $(<19 \mathrm{nM})$ and in the presence $(\sim 30 \mu \mathrm{M})$ of $\mathrm{Ca}^{2+}$, obtained using $10 \mathrm{mM}$ $\mathrm{K}_{2} \mathrm{H}_{2}$ EGTA and $\mathrm{K}_{2}$ CaEGTA buffers ${ }^{51}$ respectively (Fig. 1b). Basal GC1 activity was assessed as internal control by replacing GCAPs with equal amount of the buffer used for protein storing. Then, the enzymatic activity as a function of $\left[\mathrm{Ca}^{2+}\right]$ was measured as in previous work ${ }^{10}$, with minor changes explained in the following. Several combinations of GCAPs were tested, namely: (i) $10 \mu \mathrm{M}$ WT-GCAP1; (ii) $5 \mu \mathrm{M}$ WT-GCAP1 $+5 \mu \mathrm{M}$ E111V-GCAP1; iii) $15 \mu \mathrm{M}$ WT-GCAP1 $+5 \mu \mathrm{M}$ E111V-GCAP1. Data for E111V-GCAP1 alone are referred to our previous publication ${ }^{10}$. $\mathrm{Ca}^{2+}$ concentration in each assay was carefully determined by combining the same $\mathrm{K}_{2} \mathrm{H}_{2} \mathrm{EGTA}$ and $\mathrm{K}_{2}$ CaEGTA buffers previously mentioned. The assay consisted in incubating for $5 \mathrm{~min}$ at $\mathrm{RT}$ the diluted proteins with a fixed amount of extracted membranes, then reaction buffer $(30 \mathrm{mM}$ MOPS pH 7.2, $20 \mathrm{mM} \mathrm{KCl}, 10 \mathrm{mM}$, $4 \mathrm{mM} \mathrm{NaCl}, 1 \mathrm{mM}$ DTT, $3.5 \mathrm{mM} \mathrm{MgCl}_{2}, 1 \mathrm{mM} \mathrm{GTP}, 300 \mu \mathrm{M}$ ATP, $160 \mu \mathrm{M}$ Zaprinast) was added and the samples were incubated for $5 \mathrm{~min}$ at $30^{\circ} \mathrm{C}$. The reaction was blocked by the addition of $50 \mathrm{mM}$ EDTA and incubating at $98^{\circ} \mathrm{C}$ for $5 \mathrm{~min}$. After centrifugation at $13000 \mathrm{rpm}$ the supernatant was loaded in a C18 reverse phase column (Chromolith column, Millipore) previously equilibrated with $5 \mathrm{mM} \mathrm{KH}_{2} \mathrm{PO}_{4}$. Following a previously established protocol ${ }^{10}$ on an HPLC apparatus (Jasco) the cGMP concentration was determined by acetonitrile gradient. Four to 6 repetitions of each assay were performed. Data have been normalized to the total protein content present in membranes used for the assay (amido black, Sigma) and on the minimum and maximum GC-activity. $\mathrm{IC}_{50}$ and Hill coefficient $\left(h_{c}\right)$ values were determined for each assay by curve fitting to a 4-parameter Hill function (Sigma Plot 12.5) and mean, standard deviation and standard error of the mean (s.e.m.) were determined. All data distributions passed the Shapiro-Wilk normality test and the observed parameters $\mathrm{IC}_{50}$ and $\mathrm{h}_{\mathrm{c}}$ were compared with one another by one-tailed t-test (SigmaPlot 12.5), rejecting in all cases the null hypothesis $(\mathrm{p}<0.05)$.

Numerical simulations of mouse photoresponses. A previously developed comprehensive biochemical model of phototransduction in mouse rods was used for numerical simulations ${ }^{37}$. All parameters describing the phototransduction cascade were left unaltered, the only difference being the way the reaction describing the dynamic synthesis of cGMP by GC1 was implemented. In the present implementation, to account for specific contributions of GCAP variants to the cGMP synthesis in a mouse photoreceptor, each variant $\left(\mathrm{GCAP}_{\mathrm{i}}\right)$ was described to contribute by a specific fraction $f_{i}$ to the maximal activation $\left(\alpha_{\max }\right)$ of GC1:

$$
\frac{d[c G M P]}{d t}=\sum_{i} \frac{f_{i} \times \alpha_{\max }}{1+\left(\frac{C a_{\text {free }}^{2+}}{I C_{50_{i}}}\right)^{h_{c_{i}}}} \text { where } \sum_{i} f_{i}=1
$$

The value for $\alpha_{\max }$, assumed to be the same for each variant, was fixed to $120 \mu \mathrm{M} / \mathrm{s}$, as in the previous model, while $I C_{50}$ and $h_{c}$ for WT-GCAP1 and GCAP2 were fixed to their experimentally measured values in mouse rod outer segments ${ }^{c}$. I $C_{50}$ and $h_{c}$ for E111V-GCAP1 were set to their relative values (-fold variation) compared to the wild type, according to the parameters experimentally measured for the human orthologs (Table 1). The use of different weight factors $f_{i}$ is a convenient way to account for the specific contribution of each GCAP variant to the overall regulation of GC1, with the assumption of a similar apparent affinity between GCAP variants and GC1, so that the variants may compete for the target based solely on their concentration/expression levels. This condition has been demonstrated to be realistic for E111V and WT human GCAP1, for which substantially unaltered $\mathrm{EC}_{50}$ values for human $\mathrm{GC1}$ were measured ${ }^{10}$, as well as for murine GCAP1 and GCAP2, which showed almost identical $\mathrm{EC}_{50}$ values for murine $\mathrm{GC1}$ in rod outer segment membranes ${ }^{7}$.

With the above assumptions, by tuning the $f_{i}$ terms one can simulate a broad variety of experimental conditions; for example, the situation of a heterozygous mouse E111V/WT, corresponding to the human adCORD case, 
in which half of the GCAP1 molecules are WT and half carry the E111V mutations, in the presence of normal amounts of WT GCAP2 was modeled as follows:

$$
\begin{aligned}
\frac{d[c G M P]}{d t}= & \frac{d[c G M P]^{G C A P 1^{W T}}}{d t}+\frac{d[c G M P]^{G C A P 1^{E 111 V}}}{d t}+\frac{d[c G M P]^{G C A P 2^{W T}}}{d t} \\
= & \alpha_{\max } \times\left(\frac{0.25}{1+\left(\frac{C a_{\text {free }}^{2+}}{I C_{50}{ }_{G C A P 1} W T}\right)^{h}{ }_{G C A P 1}^{W T}}+\frac{0.25}{1+\left(\frac{C a_{\text {free }}^{2+}}{I C_{50}{ }_{G C A P 1}^{E 111 V}}\right)^{h}}\right) \\
& \left.+\frac{0.5}{1+\left(\frac{C a_{\text {free }}^{2+}}{I C_{50}{ }_{G C A P 1^{W T}}{ }^{E 111 V}}\right)^{h}}\right)
\end{aligned}
$$

The case of homozygous mouse $\mathrm{E} 111 \mathrm{~V} / \mathrm{E} 111 \mathrm{~V}$ in the presence of endogenous GCAP2 was simulated by setting $\mathrm{f}_{\mathrm{GCAP} 1}{ }_{\mathrm{WT}}=0 ; \mathrm{f}_{\mathrm{GCAP} 1}{ }^{\mathrm{E} 111 \mathrm{~V}}=0.5 ; \mathrm{f}_{\mathrm{GCAP} 2}=0.5$. Finally, the case of 3 -fold excess of WT-GCAP1 over a background of E111V-GCAP1 and endogenous GCAP2 was obtained by setting: $\mathrm{f}_{\mathrm{GCAP} 1}{ }^{\mathrm{WT}}=0.375 ; \mathrm{f}_{\mathrm{GCAP} 1}{ }^{\mathrm{E} 111 \mathrm{~V}}=0.125$; $\mathrm{f}_{\mathrm{GCAP} 2}=0.5$, while the same excess in the absence of endogenous GCAP2 was simulated by: $\mathrm{f}_{\mathrm{GCAP} 1}{ }^{\mathrm{WT}}=0.75$; $\mathrm{f}_{\mathrm{GCAP} 1}{ }^{\mathrm{E} 111 \mathrm{~V}}=0.25 ; \mathrm{f}_{\mathrm{GCAP} 2}=0$. All numerical simulations were performed in Matlab as explained in earlier works $^{36,37}$.

Received: 17 September 2019; Accepted: 12 December 2019;

Published online: 27 December 2019

\section{References}

1. Pugh, E. J. \& Lamb, T. In Handbook of Biological Physics Vol. 3 (eds DG Stavenga, WJ DeGrip, \& EN Jr Pugh) 183-255 (Elsevier Science BV, 2000).

2. Koch, K. W. \& Dell'Orco, D. Protein and Signaling Networks in Vertebrate Photoreceptor Cells. Front Mol Neurosci 8, 67, https://doi. org/10.3389/fnmol.2015.00067 (2015).

3. Koch, K. W. \& Dell'Orco, D. A calcium-relay mechanism in vertebrate phototransduction. ACS Chem Neurosci 4, 909-917, https:// doi.org/10.1021/cn400027z (2013).

4. Koch, K. W., Duda, T. \& Sharma, R. K. Ca(2+)-modulated vision-linked ROS-GC guanylate cyclase transduction machinery. Mol Cell Biochem 334, 105-115, https://doi.org/10.1007/s11010-009-0330-z (2010).

5. Sharon, D., Wimberg, H., Kinarty, Y. \& Koch, K. W. Genotype-functional-phenotype correlations in photoreceptor guanylate cyclase (GC-E) encoded by GUCY2D. Prog Retin Eye Res 63, 69-91, https://doi.org/10.1016/j.preteyeres.2017.10.003 (2018).

6. Korenbrot, J. I. Speed, sensitivity, and stability of the light response in rod and cone photoreceptors: facts and models. Prog Retin Eye Res 31, 442-466, doi:S1350-9462(12)00039-0 [pii], 10. 1016/j.preteyeres.2012.05.002 (2012).

7. Peshenko, I. V. et al. Enzymatic properties and regulation of the native isozymes of retinal membrane guanylyl cyclase (RetGC) from mouse photoreceptors. Biochemistry 50, 5590-5600, https://doi.org/10.1021/bi200491b (2011).

8. Hwang, J. Y. \& Koch, K. W. Calcium- and myristoyl-dependent properties of guanylate cyclase-activating protein-1 and protein-2. Biochemistry 41, 13021-13028, doi:bi026618y [pii] (2002).

9. Hwang, J. Y. et al. Regulatory modes of rod outer segment membrane guanylate cyclase differ in catalytic efficiency and $\mathrm{Ca}(2+)$ sensitivity. Eur J Biochem 270, 3814-3821, doi:3770 [pii] (2003).

10. Marino, V. et al. A novel p.(Glu111Val) missense mutation in GUCA1A associated with cone-rod dystrophy leads to impaired calcium sensing and perturbed second messenger homeostasis in photoreceptors. Hum Mol Genet 27, 4204-4217, https://doi. org $/ 10.1093 / \mathrm{hmg} / \mathrm{ddy} 311$ (2018).

11. Peshenko, I. V. et al. A G86R mutation in the calcium-sensor protein GCAP1 alters regulation of retinal guanylyl cyclase and causes dominant cone-rod degeneration. J Biol Chem 294, 3476-3488, https://doi.org/10.1074/jbc.RA118.006180 (2019).

12. Dell'Orco, D., Sulmann, S., Linse, S. \& Koch, K. W. Dynamics of conformational Ca2+-switches in signaling networks detected by a planar plasmonic device. Anal Chem 84, 2982-2989, https://doi.org/10.1021/ac300213j (2012).

13. Marino, V., Sulmann, S., Koch, K. W. \& Dell'Orco, D. Structural effects of $\mathrm{Mg} 2+$ on the regulatory states of three neuronal calcium sensors operating in vertebrate phototransduction. Biochim Biophys Acta 1853, 2055-2065, https://doi.org/10.1016/j. bbamcr.2014.10.026 (2015).

14. Peshenko, I. V. \& Dizhoor, A. M. Guanylyl cyclase-activating proteins (GCAPs) are Ca2+/Mg2+ sensors: implications for photoreceptor guanylyl cyclase (RetGC) regulation in mammalian photoreceptors. J Biol Chem 279, 16903-16906, https://doi. org/10.1074/jbc.C400065200C400065200[pii] (2004).

15. Peshenko, I. V. \& Dizhoor, A. M. $\mathrm{Ca}^{2+}$ and $\mathrm{Mg}^{2+}$ binding properties of GCAP-1. Evidence that $\mathrm{Mg} 2+$-bound form is the physiological activator of photoreceptor guanylyl cyclase. J Biol Chem 281, 23830-23841, doi:M600257200 [pii] 10.1074/jbc. M600257200 (2006).

16. Dizhoor, A. M., Olshevskaya, E. V. \& Peshenko, I. V. Mg2+/Ca2+ cation binding cycle of guanylyl cyclase activating proteins (GCAPs): role in regulation of photoreceptor guanylyl cyclase. Mol Cell Biochem 334, 117-124, https://doi.org/10.1007/s11010-0090328-6 (2010).

17. Behnen, P., Dell'Orco, D. \& Koch, K. W. Involvement of the calcium sensor GCAP1 in hereditary cone dystrophies. Biol Chem 391, 631-637, https://doi.org/10.1515/BC.2010.063 (2010).

18. Jiang, L. \& Baehr, W. GCAP1 mutations associated with autosomal dominant cone dystrophy. Adv Exp Med Biol 664, 273-282, https://doi.org/10.1007/978-1-4419-1399-9_31 (2010).

19. Manes, G. et al. Cone dystrophy or macular dystrophy associated with novel autosomal dominant GUCA1A mutations. Mol Vis 23, 198-209 (2017).

20. Dell'Orco, D., Behnen, P., Linse, S. \& Koch, K. W. Calcium binding, structural stability and guanylate cyclase activation in GCAP1 variants associated with human cone dystrophy. Cell Mol Life Sci 67, 973-984, https://doi.org/10.1007/s00018-009-0243-8 (2010).

21. Dizhoor, A. M., Boikov, S. G. \& Olshevskaya, E. V. Constitutive activation of photoreceptor guanylate cyclase by Y99C mutant of GCAP-1. Possible role in causing human autosomal dominant cone degeneration. J Biol Chem 273, 17311-17314 (1998). 
22. Kitiratschky, V. B. et al. Mutations in the GUCA1A gene involved in hereditary cone dystrophies impair calcium-mediated regulation of guanylate cyclase. Hum Mutat 30, E782-796, https://doi.org/10.1002/humu.21055 (2009).

23. Marino, V., Scholten, A., Koch, K. W. \& Dell'Orco, D. Two retinal dystrophy-associated missense mutations in GUCA1A with distinct molecular properties result in a similar aberrant regulation of the retinal guanylate cyclase. Hum Mol Genet 24, 6653-6666, https://doi.org/10.1093/hmg/ddv370 (2015).

24. Payne, A. M. et al. A mutation in guanylate cyclase activator 1A (GUCA1A) in an autosomal dominant cone dystrophy pedigree mapping to a new locus on chromosome 6p21.1. Hum Mol Genet 7, 273-277, doi:ddb036 [pii] (1998).

25. Vocke, F. et al. Dysfunction of cGMP signalling in photoreceptors by a macular dystrophy-related mutation in the calcium sensor GCAP1. Hum Mol Genet 26, 133-144, https://doi.org/10.1093/hmg/ddw374 (2017).

26. Olshevskaya, E. V. et al. The Y99C mutation in guanylyl cyclase-activating protein 1 increases intracellular $\mathrm{Ca}^{2+}$ and causes photoreceptor degeneration in transgenic mice. J Neurosci 24, 6078-6085, https://doi.org/10.1523/JNEUROSCI.096304.200424/27/6078[pii] (2004).

27. Buch, P. K. et al. Dominant cone-rod dystrophy: a mouse model generated by gene targeting of the GCAP1/Gucala gene. PLoS One 6, e18089, https://doi.org/10.1371/journal.pone.0018089 (2011).

28. Jiang, L. et al. RNAi-mediated gene suppression in a GCAP1(L151F) cone-rod dystrophy mouse model. PLoS One 8, e57676, https:// doi.org/10.1371/journal.pone.0057676 (2013).

29. Duda, T. et al. The calcium-sensor guanylate cyclase activating protein type 2 specific site in rod outer segment membrane guanylate cyclase type 1. Biochemistry 44, 7336-7345, https://doi.org/10.1021/bi050068x (2005).

30. Lange, C., Duda, T., Beyermann, M., Sharma, R. K. \& Koch, K. W. Regions in vertebrate photoreceptor guanylyl cyclase ROS-GC1 involved in $\mathrm{Ca}(2+)$-dependent regulation by guanylyl cyclase-activating protein GCAP-1. FEBS Lett 460, 27-31 (1999).

31. Rehkamp, A. et al. Molecular Details of Retinal Guanylyl Cyclase 1/GCAP-2 Interaction. Front Mol Neurosci 11, 330, https://doi. org/10.3389/fnmol.2018.00330 (2018).

32. Laura, R. P. \& Hurley, J. B. The kinase homology domain of retinal guanylyl cyclases 1 and 2 specifies the affinity and cooperativity of interaction with guanylyl cyclase activating protein-2. Biochemistry 37, 11264-11271, https://doi.org/10.1021/bi9809674 (1998).

33. Peshenko, I. V., Olshevskaya, E. V. \& Dizhoor, A. M. Evaluating the role of retinal membrane guanylyl cyclase 1 (RetGC1) domains in binding guanylyl cyclase-activating proteins (GCAPs). J Biol Chem 290, 6913-6924, https://doi.org/10.1074/jbc.M114.629642 (2015).

34. Vinberg, F., Peshenko, I. V., Chen, J., Dizhoor, A. M. \& Kefalov, V. J. Guanylate cyclase-activating protein 2 contributes to phototransduction and light adaptation in mouse cone photoreceptors. J Biol Chem 293, 7457-7465, https://doi.org/10.1074/jbc. RA117.001574 (2018).

35. Makino, C. L. et al. Enzymatic relay mechanism stimulates cyclic GMP synthesis in rod photoresponse: biochemical and physiological study in guanylyl cyclase activating protein 1 knockout mice. PLoS One 7, e47637, https://doi.org/10.1371/journal. pone.0047637PONE-D-12-25181[pii] (2012).

36. Dell'Orco, D., Schmidt, H., Mariani, S. \& Fanelli, F. Network-level analysis of light adaptation in rod cells under normal and altered conditions. Mol Biosyst 5, 1232-1246, https://doi.org/10.1039/b908123b (2009).

37. Invergo, B. M., Dell'Orco, D., Montanucci, L., Koch, K. W. \& Bertranpetit, J. A comprehensive model of the phototransduction cascade in mouse rod cells. Mol Biosyst 10, 1481-1489, https://doi.org/10.1039/c3mb70584f (2014).

38. Koch, K. W. \& Stryer, L. Highly cooperative feedback control of retinal rod guanylate cyclase by calcium ions. Nature 334, 64-66, https://doi.org/10.1038/334064a0 (1988).

39. Burns, M. E., Mendez, A., Chen, J. \& Baylor, D. A. Dynamics of cyclic GMP synthesis in retinal rods. Neuron 36, 81-91, https://doi. org/10.1016/s0896-6273(02)00911-x (2002).

40. Viviano, J., Krishnan, A., Wu, H. \& Venkataraman, V. Electrophoretic mobility shift in native gels indicates calcium-dependent structural changes of neuronal calcium sensor proteins. Anal Biochem 494, 93-100, https://doi.org/10.1016/j.ab.2015.11.005 (2016).

41. Otto-Bruc, A. et al. Localization of guanylate cyclase-activating protein 2 in mammalian retinas. Proc Natl Acad Sci USA 94, 4727-4732, https://doi.org/10.1073/pnas.94.9.4727 (1997).

42. Lopez-del Hoyo, N. et al. Overexpression of guanylate cyclase activating protein 2 in rod photoreceptors in vivo leads to morphological changes at the synaptic ribbon. PLoS One 7, e42994, https://doi.org/10.1371/journal.pone.0042994 (2012).

43. Lopez-Begines, S., Plana-Bonamaiso, A. \& Mendez, A. Molecular determinants of Guanylate Cyclase Activating Protein subcellular distribution in photoreceptor cells of the retina. Sci Rep 8, 2903, https://doi.org/10.1038/s41598-018-20893-1 (2018).

44. Mendez, A. et al. Role of guanylate cyclase-activating proteins (GCAPs) in setting the flash sensitivity of rod photoreceptors. Proc Natl Acad Sci USA 98, 9948-9953, https://doi.org/10.1073/pnas.171308998171308998[pii] (2001).

45. Lim, S. et al. Retinal guanylyl cyclase activating protein 1 forms a functional dimer. PLoS One 13, e0193947, https://doi.org/10.1371/ journal.pone.0193947 (2018).

46. Dell'Orco, D., Sulmann, S., Zagel, P., Marino, V. \& Koch, K. W. Impact of cone dystrophy-related mutations in GCAP1 on a kinetic model of phototransduction. Cell Mol Life Sci 71, 3829-3840, https://doi.org/10.1007/s00018-014-1593-4 (2014).

47. Kulkarni, M., Trifunovic, D., Schubert, T., Euler, T. \& Paquet-Durand, F. Calcium dynamics change in degenerating cone photoreceptors. Hum Mol Genet 25, 3729-3740, https://doi.org/10.1093/hmg/ddw219 (2016).

48. Power, M. et al. Cellular mechanisms of hereditary photoreceptor degeneration - Focus on cGMP. Prog Retin Eye Res, 100772, https://doi.org/10.1016/j.preteyeres.2019.07.005 (2019).

49. Asteriti, S. et al. Effective delivery of recombinant proteins to rod photoreceptors via lipid nanovesicles. Biochem Biophys Res Commun 461, 665-670, https://doi.org/10.1016/j.bbrc.2015.04.088 (2015).

50. Marino, V., Borsatto, A., Vocke, F., Koch, K. W. \& Dell'Orco, D. CaF2 nanoparticles as surface carriers of GCAP1, a calcium sensor protein involved in retinal dystrophies. Nanoscale 9, 11773-11784, https://doi.org/10.1039/c7nr03288a (2017).

51. Zagel, P., Dell'Orco, D. \& Koch, K. W. The dimerization domain in outer segment guanylate cyclase is a $\mathrm{Ca}(2)(+)$-sensitive control switch module. Biochemistry 52, 5065-5074, https://doi.org/10.1021/bi400288p (2013).

\section{Acknowledgements}

This work was funded by a grant from Fondazione Telethon-Italy (grant no. GGP16010 to DDO).We would like to thank Dr. Valerio Marino for helpful discussions and Anna Avesani for assistence with GCAP2 preparation.

\section{Author contributions}

D.D.O. conceived the work, performed in silico simulations and wrote the manuscript. G.D.C. designed and performed all experimental assays and contributed in writing the manuscript.

\section{Competing interests}

The authors declare no competing interests. 


\section{Additional information}

Supplementary information is available for this paper at https://doi.org/10.1038/s41598-019-56606-5.

Correspondence and requests for materials should be addressed to D.D.O.

Reprints and permissions information is available at www.nature.com/reprints.

Publisher's note Springer Nature remains neutral with regard to jurisdictional claims in published maps and institutional affiliations.

(c) (i) Open Access This article is licensed under a Creative Commons Attribution 4.0 International License, which permits use, sharing, adaptation, distribution and reproduction in any medium or format, as long as you give appropriate credit to the original author(s) and the source, provide a link to the Creative Commons license, and indicate if changes were made. The images or other third party material in this article are included in the article's Creative Commons license, unless indicated otherwise in a credit line to the material. If material is not included in the article's Creative Commons license and your intended use is not permitted by statutory regulation or exceeds the permitted use, you will need to obtain permission directly from the copyright holder. To view a copy of this license, visit http://creativecommons.org/licenses/by/4.0/.

(c) The Author(s) 2019 